\title{
Cost-effectiveness analysis of the addition of bevacizumab to temozolomide therapy for the treatment of unresected glioblastoma
}

\author{
ZHAOYAN CHEN, MEI ZHAN, FANGYUAN TIAN and TING XU \\ Department of Pharmacy, West China Hospital, Sichuan University, Chengdu, Sichuan 610041, P.R. China
}

Received March 29, 2019; Accepted September 30, 2019

DOI: $10.3892 / \mathrm{ol} .2019 .11099$

\begin{abstract}
Glioblastoma, a cancer that originates from astrocytes, is the most prevalent malignant glioma in the adult population. The aim of the present study was to evaluate the cost-effectiveness of bevacizumab (BEV) as a supplement to standard temozolomide (TMZ) treatment for unresected glioblastoma. The analyzed data were from a phase II trial that showed a survival benefit following combination therapy, when compared with TMZ monotherapy. According to the clinical symptoms and disease progression, a Markov model was constructed to estimate the incremental cost-effectiveness ratio (ICER) from a Chinese societal perspective. Health outcomes were retrieved from the GENOM 009 trial, and utility parameters were obtained from published literature. Uncertainties within the model were addressed through one-way deterministic and probabilistic sensitivity analyses. The addition of BEV to TMZ therapy increased overall costs by $\$ 30,894.99$, with a gain of 0.18 quality-adjusted life-years (QALYs), resulting in an ICER of \$171,638.83/QALY. Both one-way sensitivity and probabilistic sensitivity analyses confirmed that BEV/TMZ co-treatment was not cost-effective in the context of a $\$ 26,508.00 / \mathrm{QALY}$ willingness-to-pay (WTP) threshold. The utility of the progression-free survival state had the most noticeable impact on the ICER. In summary, the combination of BEV and TMZ should not be considered a cost-effective neoadjuvant treatment option for patients with unresected glioblastoma in China, from a societal perspective. However, in view of the survival benefits conferred, an appropriate price discount or the use of medical insurance could make BEV affordable for this patient population.
\end{abstract}

Correspondence to: Professor Ting Xu, Department of Pharmacy, West China Hospital, Sichuan University, 37 Guoxue Alley, Wuho, Chengdu, Sichuan 610041, P.R. China

E-mail: tingx2009@163.com

Key words: cost-effectiveness, unresected glioblastoma, bevacizumab, neoadjuvant treatment

\section{Introduction}

Gliomas are common malignant brain tumors, with an incidence of 3-4 new cases per 100,000 adults each year worldwide (1). As a result of the aggressive nature of gliomas, prognosis is poor; the median survival time for patients is $\sim 1$ year, with $<10 \%$ of patients surviving $>3$ years post-diagnosis (2). The standard treatment for glioblastoma is radiotherapy alongside temozolomide (TMZ) chemotherapy. However, its efficacy is limited; even with treatment, the median survival time of patients with gliomas is 12-18 months (3).

Bevacizumab (BEV) inhibits tumor growth by binding to vascular endothelial growth factor and preventing its interaction with receptors on the endothelial cell surface. Survival data from the GENOM 009 trial (a randomized phase II trial), which compared TMZ treatment to TMZ/BEV combination therapy in adult patients with unresected glioblastoma, are currently used for cost-effective analysis (4). The trial results indicated that both progression-free survival (PFS; 4.8 months vs. 2.2 months) and overall survival (OS; 10.6 months vs. 7.7 months) were longer in patients treated with combination of BEV and TMZ (4). Although this increase in survival was not statistically significant, these clinical data may provide a new option for patients and policy makers in the treatment of unresected glioblastoma.

Considering the efficacy and high cost of BEV, it was included in the National Health Insurance Directory (2017) following negotiations between the government and pharmaceutical companies. Thus, the retail price of BEV $(100 \mathrm{mg} / 4 \mathrm{ml})$ was reduced from $\$ 852.23 /$ unit to $\$ 305.38$ /unit in Sichuan, a $64.17 \%$ reduction. Considering the efficacy of BEV in glioma treatment, the rapidly rising cost of drugs and the current lack of medical resources, a cost-effectiveness analysis would be useful to measure the potential economic benefit of BEV/TMZ co-treatment of patients with glioblastoma, particularly in China (a developing country). In the current study, a Markov model was used to estimate the cost-effectiveness of BEV/TMZ co-therapy as a neoadjuvant treatment option for patients with glioblastoma.

\section{Materials and methods}

Patients and therapy. The clinical data used in the present study were retrieved from the GENOM 009 trial (4), a phase II 
study in which 102 patients, including 54\% (55/102) male and $46 \%$ (47/102) female, (mean, 62; range, 36-75 years) with unresected glioblastoma were randomized to either the TMZ control arm or the TMZ+BEV treatment arm (ratio, 1:1). The inclusion criteria included: i) Patients with unresected glioblastoma; ii) diagnosis using biopsy (including stereotactic or open craniotomy); iii) no prior treatment; iv) a tumor size $\geq 2 \mathrm{~cm}$; and v) exhibiting stable or decreasing glucocorticoid doses within 5 days of randomization. Moreover, in patients undergoing craniotomy, post-operative magnetic resonance imaging (MRI) was mandatory within $72 \mathrm{~h}$. Other inclusion criteria including: Age $\geq 18$ years, Eastern Cooperative Oncology Group performance status $0-2$, Barthel index $\geq 50 \%$, adequate healing of craniotomy or cranial biopsy (infection or bleeding at the wound site), normal baseline hematology and biochemistry, and the absence of proteinuria. The exclusion criteria were: i) Patient history of a prior malignant infiltrating disease during the last five years; ii) uncontrolled arterial hypertension; iii) inflammatory digestive disease; iv) cardiac or vascular disease; and v) recent symptomatic intracranial hemorrhage discovered using post-operative MRI or post-biopsy computerized tomography. Patients were randomly assigned to receive $\mathrm{BEV}(10 \mathrm{mg} / \mathrm{kg} / \mathrm{day}$; days 1 and 15; administrated $I V$ drip) and TMZ (85 mg/m²/day; days $1-21$; administrated per $o s$ ), or TMZ alone ( $85 \mathrm{mg} / \mathrm{m}^{2} /$ day; days $1-21$; administrated per os) for two 28-day cycles. After a 28-day break, BEV maintenance was allowed in the BEV + TMZ group for a maximum of six cycles, until the disease progressed or unacceptable toxic effects developed.

Model structure. A Markov decision tree model was selected to compare the clinical and economic data associated with the two therapeutic strategies (Fig. 1). The model is composed of mutually exclusive disease states, including progression-free survival (PFS), progressive disease (PD) and death. All patients with unresected glioblastoma begin treatment in the PFS state, but they may move between health states over time (Fig. 1 and 2). Following a Markov cycle, patients in the PFS state may transition to the PD or death state, or may remain in the PFS state. However, patients in the PD state cannot return to the PFS state, and all patients may transition to the death state (Fig. 2). For patients with unresected tumors, a poor prognosis is common, with no significant difference in overall survival between treatment with standard chemoradiotherapy, and radiotherapy alone ( 9.4 months vs. 7.8 months; hazard ratio [HR], $0.7,95 \% \mathrm{CI} 0.5-0.9)(5)$. The absence of previous debulking surgery increases the likelihood of neurological instability during treatment (5). According to the clinical symptoms and progression time of the disease, the transition cycle length was adjusted to one month in the current study. Monthly transition probabilities were converted from median survival estimates (Table I) using the following formula: $\mathrm{P}(1$ month $)=1-(0.5)^{\wedge}(1 /$ median time to event $)$, which was derived through the formula $\mathrm{P}=1-\mathrm{e}-\mathrm{R}$, where $\mathrm{R}=-\ln [0.5] /$ (time to event/number of treatment cycles) $(6,7)$. The time horizon chosen for this model was 10 years, at which timepoint all patients had succumbed to the disease.

Model parameters. Costs were estimated from a Chinese societal perspective (Table II). The following costs were considered during analysis: Anticancer drugs, tests (enhanced head CT, blood biochemical examination), management of grade 3-4 adverse events (AEs), absenteeism and hospitalization. Implicit costs were ignored as a consequence of individual differences. The dosage of anticancer drugs was calculated based on the median reported body surface area and weight in China (8). Unit price for each drug and test was obtained by consulting the 2017 fee standards of West China Hospital, Sichuan University. Regarding the data retrieved from the GENOM 009 trial, the cost of second-line treatment in the two groups was estimated. In the control group, TMZ was administered orally in the outpatient department, whilst patients in the treatment group needed to be hospitalized for intravenous administration. Consequently, loss-of-productivity was estimated to be higher in the treatment group. The present study estimated the cost of absenteeism by referring to the gross domestic product (GDP) per capita of Sichuan in 2017 (\$7,057.26/person/year) (9). All costs were converted to US dollars, with an exchange rate of $\$ 1=¥ 6.3339$ (Jan, 2018) (10).

Data on health outcomes in the present model were derived from the GENOM 009 study. Survival time was adjusted to quality-adjusted life years (QALY) using both utility scores and transition probabilities of the health state. Given that no information on utilities was available in the original literature, health utility values were acquired from a study that used the standard gamble to evaluate glioblastoma health states, based on a small section of the general population of the United Kingdom (11) Consequently, the utility values for the progression-free disease, progressive disease, and death states were $0.89,0.73$ and 0.00 , respectively. Model parameters related to costs and effectiveness, which were derived from the GENOM 009 trial, are depicted in Table II. In view of the recommendations of the 2015 China Guidelines for Pharmacoeconomic Evaluations and Manual (12), both the costs and the utility values were discounted at an annual rate of $3 \%$.

Sensitivity analyses. All parameters used in the evaluation (excluding discount rate) varied in the results of the deterministic one-way sensitivity analysis (range $= \pm 20 \%$ ). The discount rate ranged between 0 and $8 \%$. Probabilistic sensitivity analysis was conducted using a second-order Monte Carlo simulation by running 1,000 iterations (13). Cost-effectiveness acceptability analysis was conducted to evaluate optimal strategies at various willingness-to-pay (WTP) thresholds. The WTP threshold was set as three times the GDP per capita in 2017, which was \$26,508.00/QALY in China (14).

\section{Results}

Cost-effectiveness analysis. Over a 10-year time horizon, the addition of BEV to TMZ treatment increased the total QALYs by 0.18 (0.71 vs. 0.53 QALYs). However, total costs in the BEV+TMZ group were significantly higher than in the TMZ group $(\$ 50,190.52$ vs. $\$ 19,295.53)$. Therefore, the co-administration of BEV had an incremental cost-effectiveness ratio (ICER) of $\$ 171,638.83 / \mathrm{QALY}$ compared with $\mathrm{TMZ}$ alone, exhibiting a $0 \%$ chance of being cost effective at the WTP threshold of $\$ 26,508.00 /$ QALY (Table III). 


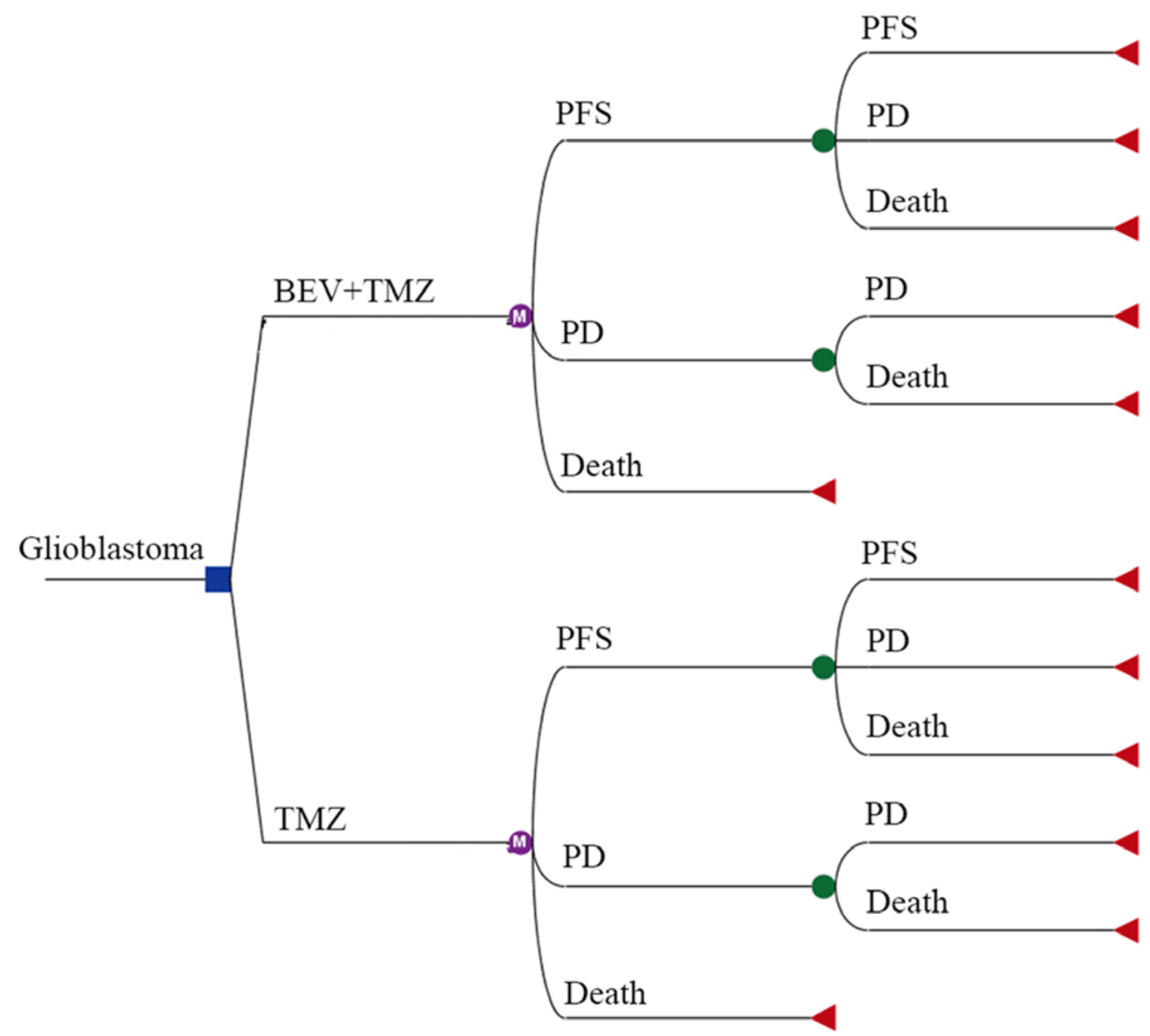

Figure 1. Markov model for unresected glioblastoma based on the GENOM 009 trial. The two groups were treated with either BEV+TMZ or TMZ alone. TMZ, temozolomide; PFS, progression-free survival; PD, progressive disease.

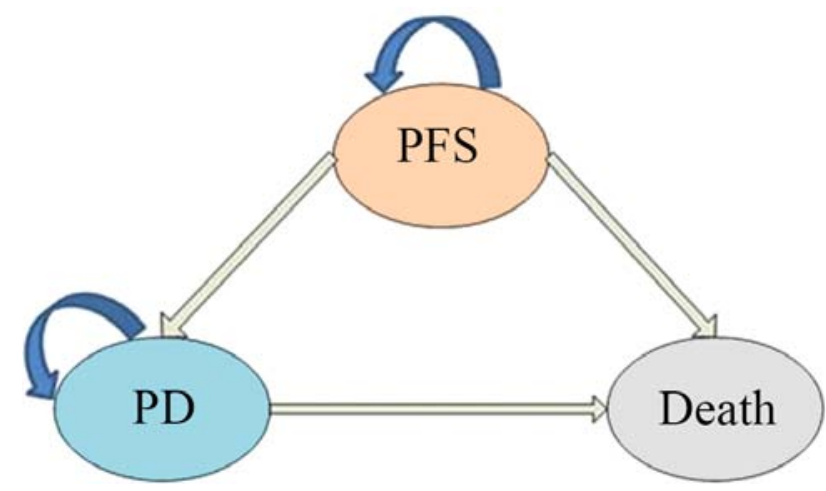

Figure 2. Markov model health states. A Markov model simulating the following three health states: PFS, PD and death. Arrows indicate staying in one state or transitioning from one state to another. PFS, progression-free survival; PD, progressive disease.

Accounting for the increase in total costs, the addition of BEV to TMZ treatment was not an economically viable treatment option for unresected glioblastoma from a Chinese societal perspective. The cost of combination treatment for patients in the PFS disease state was $>3$ times that of TMZ treatment alone $(\$ 38,211.30$ vs. $\$ 11,476.12)$. Moreover, when compared with the TMZ group, the overall costs for the PD state in the BEV+TMZ group were $\$ 4,159.81$ higher per person (\$11,979.22 vs. \$7,819.41).

\section{Sensitivity analysis}

One-way sensitivity analysis. One-way sensitivity analysis was conducted to assess the impact of individual parameters in the Markov model. The results are illustrated using a tornado diagram (Fig. 3). The utility of the PFS state, cost of $\mathrm{BEV}$ and the duration of the PFS state in the BEV+TMZ group all showed a variation of $\pm 20 \%$, and were the most influential parameters of the model. Changing the utility of PFS from 0.71 to 1.00 caused the ICER to decrease from $\$ 221,561.85$ to $\$ 155,990.85 / \mathrm{QALY}$. Variation in the cost of BEV treatment from $\$ 3,312.18 /$ month to $\$ 4,968.27 /$ month saw the ICER increase from $\$ 153,324.46 / \mathrm{QALY}$ to $\$ 197,603.80 / \mathrm{QALY}$. However, variations in the costs related to management of grade 3-4 AEs, tests used or hospital fees incurred, had a smaller impact on the ICER values predicted by sensitivity analysis. 
Table I. Transition probabilities between glioblastoma disease states.

\begin{tabular}{lccc}
\hline Transition probabilities & Baseline value & Lower limit & Upper limit \\
\hline BEV+TMZ group & & & 0.95 \\
$\mathrm{P}_{\text {PFS-PFS-1 }}$ & 0.81 & 0.11 & 0.16 \\
$\mathrm{P}_{\text {PFS-PD-1 }}$ & 0.13 & 0.05 & 0.08 \\
$\mathrm{P}_{\text {PFS-death-1 }}$ & 0.06 & 0.71 & 1.00 \\
$\mathrm{P}_{\text {PD-PD-1 }}$ & 0.89 & 0.09 & 0.14 \\
$\mathrm{P}_{\text {PD-death-1 }}$ & 0.11 & & 0.77 \\
TMZ group & & 0.51 & 0.32 \\
$\mathrm{P}_{\text {PFS-PFS-2 }}$ & 0.64 & 0.22 & 0.10 \\
$\mathrm{P}_{\text {PFS-PD-2 }}$ & 0.27 & 0.07 & 1.00 \\
$\mathrm{P}_{\text {PFS-death-2 }}$ & 0.09 & 0.71 & 0.14 \\
$\mathrm{P}_{\text {PD-PD-2 }}$ & 0.88 & 0.09 & \\
$\mathrm{P}_{\text {PD-death-2 }}$ & 0.12 & & \\
\hline
\end{tabular}

BEV, bevacizumab; TMZ, temozolomide; PFS, progression-free survival; PD, progressive disease.

Table II. Model parameters related to costs and effectiveness collected from the GENOM 009 trial.

\begin{tabular}{|c|c|c|}
\hline Parameter & $\mathrm{BEV}+\mathrm{TMZ}$ group & TMZ group \\
\hline \multicolumn{3}{|l|}{ Clinical efficacy, months } \\
\hline Median PFS, months (95\% CI) & $4.8(4.0-5.6)$ & $2.2(2.0-2.5)$ \\
\hline Median OS, months (95\% CI) & $10.6(6.9-14.3)$ & $7.7(5.4-10.0)$ \\
\hline \multicolumn{3}{|l|}{ Probability of grades 3-4 adverse events, $\%$} \\
\hline Hypertension & 4.17 & 0.00 \\
\hline Hemorrhage & 2.08 & 0.00 \\
\hline Gastrointestinal perforation & 4.17 & 0.00 \\
\hline Thrombocytopenia & 2.08 & 11.11 \\
\hline Leucopenia & 2.08 & 4.44 \\
\hline Febrile neutropenia & 2.08 & 4.44 \\
\hline Nausea and vomiting & 2.08 & 0.00 \\
\hline Infection & 10.42 & 6.67 \\
\hline Thrombotic events & 4.17 & 6.67 \\
\hline Asthenia & 4.17 & 2.22 \\
\hline \multicolumn{3}{|l|}{ Unit costs, $\$ /$ months } \\
\hline Bevacizumab,100 mg/4 ml & 4140.22 & 0.00 \\
\hline Temozolomide, 100 mg x 5 & $3,691.64$ & $4,798.422$ \\
\hline Cost of tests & 60.32 & 55.36 \\
\hline Hospitalization & 47.36 & 0.00 \\
\hline Absence from work & 108.35 & 78.80 \\
\hline Cost for adverse events & 68.71 & 132.98 \\
\hline Cost for the progressive disease state & $1,990.75$ & $1,282.90$ \\
\hline Annual discount rate, $\%$ & 3.00 & 3.00 \\
\hline
\end{tabular}

BEV, bevacizumab; TMZ, temozolomide; PFS, progression-free survival; PD, progressive disease; OS, overall survival.

Probabilistic sensitivity analysis. The acceptability curve revealed that the acceptable proportion of $\mathrm{BEV}$ and $\mathrm{TMZ}$ co-administration being a cost-effective treatment for this patient population in China, compared with TMZ mono- therapy, was zero, unless the WTP increased to $\$ 80,000 /$ QALY (Fig. 4). Additionally, probabilistic sensitivity analysis $(1,000$ iterations) demonstrated that the ICER was consistently greater than \$26,508.00/QALY (Fig. 5). 
Table III. Results of the cost-effectiveness analysis.

\begin{tabular}{lcc}
\hline Parameter & BEV+TMZ group & TMZ group \\
\hline Costs for the PFS state, \$ & $38,211.30$ & $11,476.12$ \\
Costs for the PD state, \$ & $11,979.22$ & $7,819.41$ \\
Total costs, \$ & $50,190.52$ & $19,295.53$ \\
Incremental costs, \$ & $30,894.99$ & $/$ \\
Effectiveness for the PFS state, QALYs & 0.35 & 0.17 \\
Effectiveness for the PD state, QALYs & 0.36 & 0.36 \\
Total effectiveness, QALYs & 0.71 & 0.53 \\
Incremental effectiveness, QALYs & 0.18 & $/$ \\
Total C/E, \$/QALY & $70,690.87$ & $36,406.66$ \\
ICER, \$/QALY & $171,638.83$ & $/$
\end{tabular}

BEV, bevacizumab; TMZ, temozolomide; PFS, progression-free survival; PD, progressive disease; QALY, quality-adjusted life year; C/E, cost-effectiveness; ICER, incremental cost-effectiveness ratio.

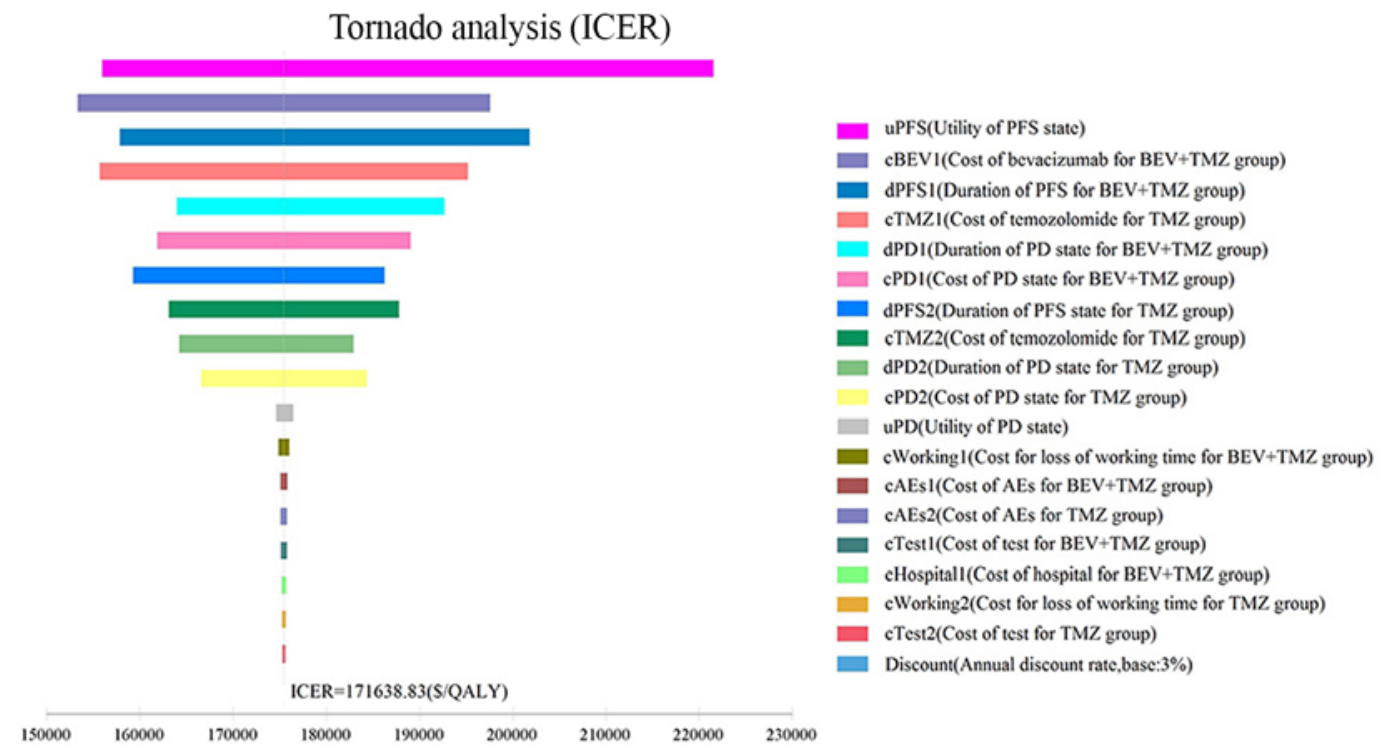

Figure 3. Tornado diagram of one-way sensitivity analysis. Tornado diagram summarizing the results of one-way sensitivity analysis to evaluate model parameters. BEV, bevacizumab; TMZ, temozolomide; PFS, progression-free survival; PD, progressive disease; AE, adverse event.

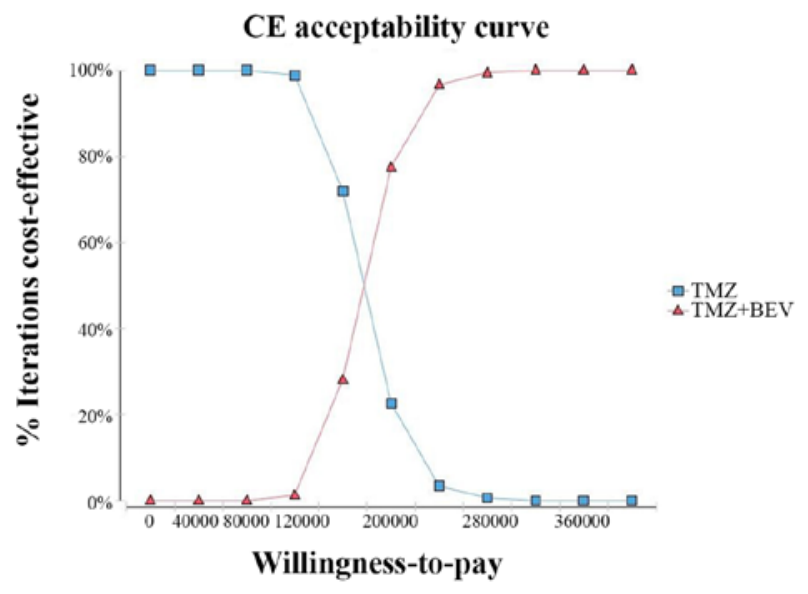

Figure 4. Cost-effectiveness acceptability curves comparing BEV and TMZ as a neoadjuvant treatment option vs. TMZ alone, at different willingness-to-pay thresholds. BEV, bevacizumab; TMZ, temozolomide; CE, cost-effectiveness.

\section{Discussion}

According to the Markov model used in the present study, the total costs were estimated at $\$ 50,190.52$ for the BEV+TMZ arm and $\$ 19,295.53$ for the TMZ arm (a difference of $\$ 30,894.99$ ). The BEV+TMZ arm exhibited a higher number of QALYs gained (0.71) compared with the TMZ arm (0.53). The ICER, or the cost per QALY gained, was $\$ 171,638.83$ in the base case analysis, which was higher than the accepted WTP threshold $(\$ 26,508.00)$ in China (15). Thus, the addition of BEV to TMZ treatment cannot be considered a cost-effective option for unresected glioblastoma, from a Chinese societal perspective.

$\mathrm{BEV}$, a monoclonal antibody, was first approved by the US Food and Drug Administration for the treatment of recurrent glioblastoma in 2009 (16). A systematic review examining the treatment of newly diagnosed glioblastoma showed that additional BEV significantly increased the median PFS time (10.6-13.6 months vs. 6.2-7.6 months) (HR, 0.74; 95\% CI, 


\section{Incremental cost-effectiveness, TMZ+BEV vs. TMZ}

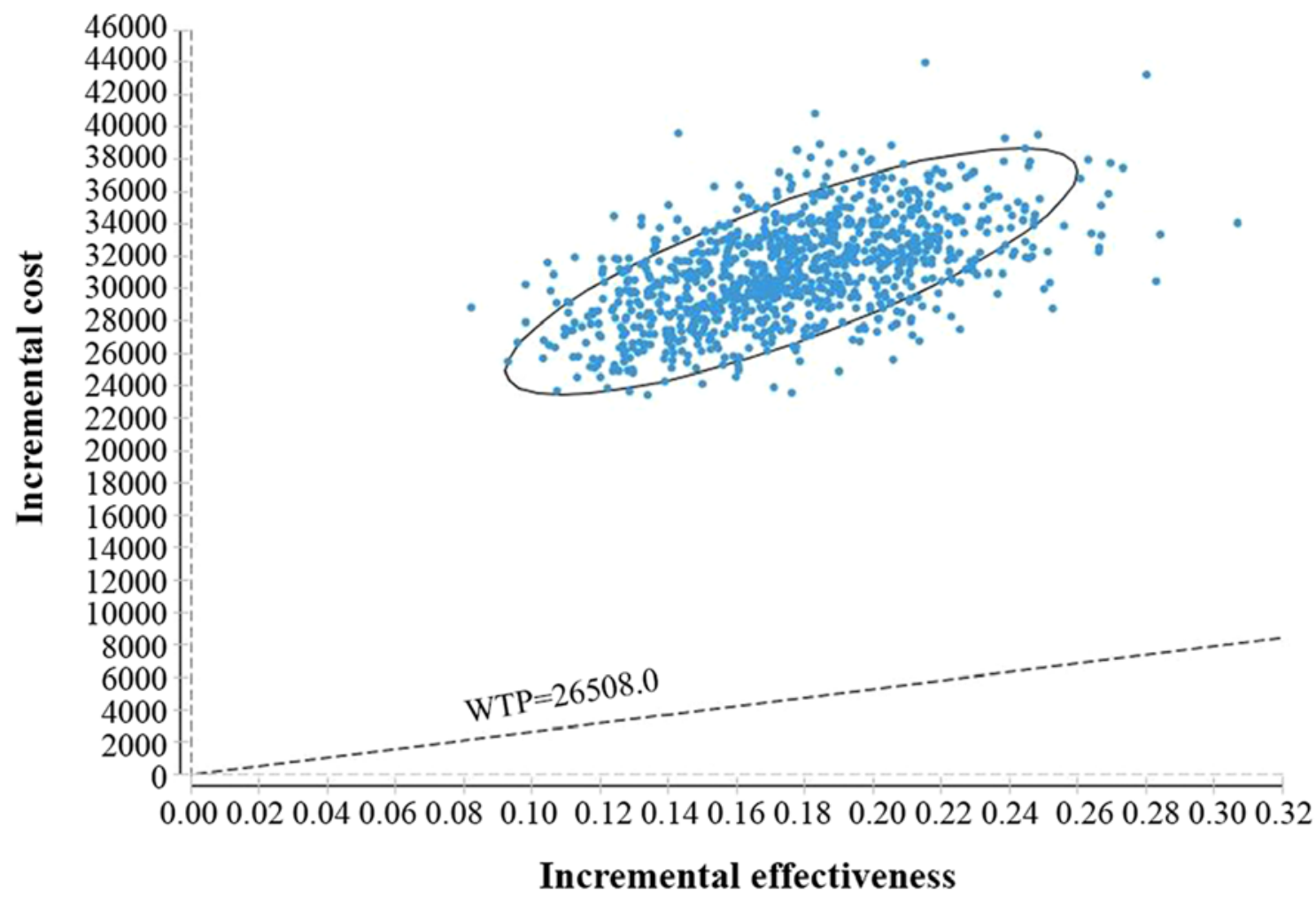

Figure 5. ICER scatter plot reflecting the variation and concentration of ICER values following probabilistic sensitivity analysis. BEV, bevacizumab; TMZ, temozolomide; WTP, willingness to pay; ICER, incremental cost-effectiveness ratio.

0.62-0.88; $\mathrm{P}=0.0009)(17)$. However, several pharmacoeconomic studies have concluded that combining BEV with other antineoplastic drugs is not cost-effective (16-18). In Canada, the ICER associated with BEV treatment was \$607,966/QALY for high-grade glioma, which far exceeded the WTP threshold of $\$ 100,000 /$ QALY, and did not fall below \$450,000/QALY in one-way sensitivity analysis (18). The estimated ICER of chemotherapy plus BEV was $\$ 295,164 /$ QALY for cervical cancer, based on a phase III randomized trial (19). Moreover, in a USA study, BEV and chemotherapy increased the mean QALYs by 0.13 for patients with advanced non-small cell lung cancer, compared with chemotherapy only. However, the ICER was $\sim 5.6$ times that of the accepted WTP threshold in the USA (20).

In accordance with the World Health Organization Guide to Generalized Cost-Effectiveness Analysis (15), the present study also determined that BEV/TMZ combination therapy was not a cost-effective treatment option for patients with unresected glioblastoma in China. In consideration of its efficacy and high cost, BEV was included in the National Health Insurance Directory (2017) following negotiations between the government and pharmaceutical companies. Prices of BEV haver remained the same. Although the price reduction did not reduce the ICER below the WTP threshold, a further price reduction, social assistance and/or medical insurance may contribute to making BEV more affordable for patients.

A cost reduction in BEV treatment would evidently improve cost-effectiveness. One-way sensitivity analysis demonstrated that the ICER increased from
$\$ 153,324.46 /$ QALY to $\$ 197,603.80 / \mathrm{QALY}$ in response to a change in BEV treatment costs from $\$ 3,312.18 /$ month to $\$ 4,968.27 /$ month. Although the cost of BEV was previously reduced by $64.17 \%$, the ICER remained markedly higher than the WTP threshold. The high cost of targeted therapies present a financial barrier to multiple treatments in the Chinese healthcare system. However, reflecting on the promising treatment improvements shown, provinces with a high GDP should consider the inclusion of BEV treatment in the local supplement list.

In the present study, a Markov decision tree model was constructed to simulate the glioblastoma disease process. However, there were some limitations: i) The cost-effectiveness analysis model was based on a phase II trial; ii) the patients were not Chinese; and iii) the treatment costs were determined using prices from Sichuan, which are subject to variation throughout the rest of China.

In summary, the results of the present study indicated that a combination of $\mathrm{BEV}$ and $\mathrm{TMZ}$ as a first-line treatment option for unresected glioblastoma, is not cost-effective from a societal perspective in China, and that the ICER is significantly higher than the WTP threshold $(\$ 26,508.00)$ in China. However, an appropriate price reduction and social assistance should be considered to ensure BEV is more affordable for this patient population.

\section{Acknowledgements}

Not applicable. 


\section{Funding}

No funding was received.

\section{Availability of data and materials}

Not applicable.

\section{Authors' contributions}

TX conceived and designed the experiments. ZYC and MZ performed the experiments. ZYC and MZ performed data analysis. ZYC and FYT provided the reagents, materials and analysis tools. ZYC wrote the manuscript. FYT revised the work critically for important intellectual content. All authors have read and approved the final version of the manuscript.

\section{Ethics approval and consent to participate}

Not applicable.

\section{Patient consent for publication}

Not applicable.

\section{Competing interests}

The authors declare that they have no competing interests.

\section{References}

1. Therese A, Dolecek, Jennifer M, Propp, Nancy E, Stroup and Carol Kruchko: CBTRUS statistical report: Primary brain and central nervous system tumors diagnosed in the united states in 2005-2009. Neuro Oncol 14 (Suppl): v1-v49, 2012.

2. Aldape KD, Okcu MF, Bondy ML and Wrensch M: Molecular epidemiology of glioblastoma. Cancer J 9: 99-106, 2003.

3. Stupp R, Mason WP, van den Bent MJ, Weller M, Fisher B, Taphoorn MJ, Belanger K, Brandes AA, Marosi C, Bogdahn U, et al: Radiotherapy plus concomitant and adjuvan temozolomide for glioblastoma. N Engl J Med 352: 987-996, 2005.

4. Balana C, De Las Penas R, Sepúlveda JM, Gil-Gil MJ, Luque R, Gallego O, Carrato C, Sanz C, Reynes G, Herrero A, et al: Bevacizumab and temozolomide versus temozolomide alone as neoadjuvant treatment in unresected glioblastoma: The GENOM 009 randomized phase II trial. J Neurooncol 127: 569-579, 2016.
5. Stupp R, Hegi ME, Mason WP, van den Bent MJ, Taphoorn MJ, Janzer RC, Ludwin SK, Allgeier A, Fisher B, Belanger K, et al: Effects of radiotherapy with concomitant and adjuvant temozolomide versus radiotherapy alone on survival in glioblastoma in a randomized phase III study: 5-Year analysis of the EORTC-NCIC trial. Lancet Oncol 10: 459-466, 2009.

6. Purmonen T, Martikainen JA, Soini EJ, Kataja V, Vuorinen RL and Kellokumpu-Lehtinen PL: Economic evaluation of sunitinib malate in second-line treatment of metastatic renal cell carcinoma in Finland. Clin Ther 30: 382-392, 2008.

7. Miller DK and Homan SM: Determining transition probabilities: Confusion and suggestions. Med Decis Making 14: 52-58, 1994.

8. Wu B, Ye M, Chen H and Shen JF: Costs of trastuzumab in combination with chemotherapy for HER2-positive advanced gastric or gastroesophageal junction cancer: An economic evaluation in the Chinese context. Clin Ther 34: 468-479, 2012.

9. http://www.sc.gov.cn/10462/12771/2018/1/31/10444117.shtml. Accessed March.15, 2018

10. http://www.boc.cn/sourcedb/whpj/. Accessed Jan 312018

11. Garside R, Pitt M, Anderson R, Rogers G, Dyer M, Mealing S, Somerville M, Price A and Stein K: The effectiveness and cost-effectiveness of carmustine implants and temozolomide for the treatment of newly diagnosed high-grade glioma: A systematic review and economic evaluation. Health Technol Assess 11: iii-iv, ix-221, 2007.

12. https://book.douban.com/subject/26922525/. Accessed Jan 312018.

13. Wu B, Miao Y, Bai Y, Ye M, Xu Y, Chen H, Shen J and Qiu Y: Subgroup economic analysis for glioblastoma in a health resource-limited setting. PLoS One 7: e34588, 2012.

14. http://www.stats.gov. cn/english/PressRelease/201801/t20180118_1574943.html. Accessed Jan 312018.

15. Murray CJ, Evans DB, Acharya A and Baltussen RM: Development of WHO guidelines on generalized cost-effectiveness analysis. Health Econ 9: 235-251, 2000.

16. Cohen MH, Shen YL, Keegan P and Pazdur R: FDA drug approval summary: Bevacizumab (Avastin) as treatment of recurrent glioblastoma multiforme. Oncologist 14: 1131-1138, 2009.

17. Fu P, He YS, Huang Q, Ding T, Cen YC, Zhao HY and Wei X: Bevacizumab treatment for newly diagnosed glioblastoma: Systematic review and meta-analysis of clinical trials. Mol Clin Oncol 4: 833-838, 2016.

18. Kovic B1 and Xie F: Economic evaluation of bevacizumab for the first-line treatment of newly diagnosed glioblastoma multiforme. J Clin Oncol 33: 2296-2302, 2015.

19. Minion LE, Bai J, Monk BJ, Robin Keller L, Ramez EN, Forde GK, Chan JK and Tewari KS: A Markov model to evaluate cost-effectiveness of antiangiogenesis therapy using bevacizumab in advanced cervical cancer. Gynecol Oncol 137: 490-496, 2015.

20. Goulart B and Ramsey S: A trial-based assessment of the cost-utility of bevacizumab and chemotherapy versus chemotherapy alone for advanced non-small cell lung cancer. Value Health 14: 836-845, 2011.

This work is licensed under a Creative Commons Attribution-NonCommercial-NoDerivatives 4.0 International (CC BY-NC-ND 4.0) License. 